\title{
A Spatial J-Test for Model Specification Against a Single or a Set of Nonnested Alternatives
}

\author{
Harry H. Kelejian ${ }^{1}$ \\ University of Maryland, College park
}

Key words: Spatial Models, Non-nested J-Test, Model Specification

\begin{abstract}
In this paper we suggest a J-type test for a given spatial model against one or more nonnested alternatives. The considered models can, but need not, contain spatial lags in both the dependent variable and disturbance term. The test is computationally simple and quite intuitive. Our suggested test is based on formal large sample results which account for triangular arrays.
\end{abstract}

\section{Introduction $^{2}$}

The J-test is a well established procedure for testing non-nested models ${ }^{3}$. Essentially, the procedure relates to whether or not the predictive value of an alternative model adds significance to the null model.

The purpose of this paper is to suggest a J-test for the specification of spatial model containing a spatially lagged dependent variable and a spatially lagged disturbance term, henceforth a $\operatorname{SARAR}(1,1)$ model, when the alternative is one, or more $\operatorname{SARAR}(1,1)$ models. Our suggested test is computationally simple, and its rationale is obvious. It is also based on formal large sample results which account for triangular arrays.

\footnotetext{
${ }^{1}$ Department of Economics, University of Maryland, College Park, MD 20742. E-mail: Kelejian@econ.umd.edu

${ }^{2}$ I would like to thank Roger Betancourt for suggesting certain problems to me involving the testing of non-nested hypotheses which then led to this paper. I would also like to thank Ingmar Prucha for useful comments on an earlier draft of this paper. Finally, I am pleased to acknowledge financial support from the National Institute of Aging/National Institue of Heath, Grant \# 2 R44 AG027622-02.

${ }^{3}$ See e.g., Davidson and MacKennen (1981), MacKennen, White, and Davidson (1983), Godfrey (1983), and the reviews given in Greene (2003, pp. 153-155, 178-180) and Kmenta (1986, pp 593-600). A nice overview of various procedures for testing nonnested hypotheses, as well as certain Monte Carlo results for doing so are given in Anselin (1986; another study of issues relating to non-nested models is given in Pesaran and Weeks (2001).
} 
Our model is specified in Section 2. Our suggested test is described in Section 3. This paper is meant to be user friendly and so all technical details, including the formal model specifications, are relegated to the appendices.

\section{The Null and Alternative Models}

The model of the null hypothesis is

$$
\begin{aligned}
y_{n} & =X_{n} \beta+\lambda W_{n} y_{n}+u_{n} \\
& =Z_{n} \gamma+u_{n} \\
u_{n} & =\rho M_{n} u_{n}+\varepsilon_{n}
\end{aligned}
$$

where $Z_{n}=\left(X_{n}, W_{n} y_{n}\right), \gamma^{\prime}=\left(\beta^{\prime}, \lambda\right), y_{n}$ is an $n \times 1$ vector of observations on the dependent variable, $X_{n}$ is an $n \times k$ matrix of observations on exogenous variables, $W_{n}$ and $M_{n}$ are $n \times n$ exogenous weighting matrices which may be equal, $u_{n}$ is an $n \times 1$ vector of disturbance terms, $\varepsilon_{n}$ is the corresponding vector of innovations, $\lambda$ and $\rho$ are scalar parameters, and $\beta$ is a $k \times 1$ parameter vector. The subscript $n$ denotes possible dependence of the matrices and vectors on the sample size $\mathrm{n}$, and so our specifications allow for triangular arrays.

We assume the researcher wishes to test the model in (1) against G alternatives. These alternative models are

$$
\begin{aligned}
y_{n} & =X_{n, i} \beta_{i}+\lambda_{i} W_{n, i} y_{n}+u_{n, i} \\
& =Z_{n, i} \gamma_{i}+u_{n, i} \\
u_{n, i} & =\rho_{i} M_{n, i} u_{n, i}+\varepsilon_{n, i}, i=1, \ldots, G
\end{aligned}
$$

where $X_{n, i}$ is an $n \times k_{i}$ matrix of observations on the exogenous variables appearing in the $i^{\text {th }}$ considered model, $G$ is a finite constant, etc. As indicated above, our formal specifications are given in the appendix. As a preview, our assumptions relating to the null model contain those in Kelejian and Prucha (1998); for future reference, we note that those assumptions include the specification that the elements of the innovation vector, $\varepsilon_{n}$, in the null model are identically distributed, and for each given sample size $n$, are i.i.d. $\left(0, \sigma_{\varepsilon}^{2}\right)$ with a finite fourth moment. Because we consider G possible alternatives to the null model, the assumptions in Kelejian and Prucha (1998) which relate to our null model are correspondingly augmented. 


\section{The Suggested J-Test}

Premultiplying the null model by $\left(I_{n}-\rho M_{n}\right)$ yields

$$
y_{n}(\rho)=Z_{n}(\rho) \gamma+\varepsilon_{n}
$$

where $y_{n}(\rho)=\left(I_{n}-\rho M_{n}\right) y_{n}$ and $Z_{n}(\rho)=\left(I_{n}-\rho M_{n}\right) Z_{n}$. Let $Z_{n, i}\left(\rho_{i}\right)=$ $\left(I_{n}-\rho_{i} M_{n, i}\right) Z_{n, i}$, and let $\hat{\gamma}_{n, i}$ be some estimator of $\gamma$ (described below). Then an evident extension of the J-test procedure to a spatial framework would be in terms of an augmented version of (3), namely

$$
\begin{aligned}
y_{n}(\rho) & =Z_{n}(\rho) \gamma+\sum_{i=1}^{G} \alpha_{i}\left[Z_{n, i}\left(\rho_{i}\right) \hat{\gamma}_{n, i}\right]+\varepsilon_{n} \\
& =Z_{n}(\rho) \gamma+\sum_{i=1}^{G} \alpha_{i}\left[Z_{n, i} \hat{\gamma}_{i}\right]+\sum_{i=1}^{G} \phi_{i}\left[M_{n, i} Z_{n, i} \hat{\gamma}_{n, i}\right]+\varepsilon_{n}
\end{aligned}
$$

where $\alpha_{i}$ is a scalar parameter, $\phi_{i}=-\alpha_{i} \rho_{i}$ and, given the null model, the true value of $\alpha_{i}=0, i=1, \ldots, G$. Let $\delta=\left(\alpha_{1}, \ldots, \alpha_{G}, \phi_{1}, \ldots, \phi_{G}\right)^{\prime}$. Clearly a test of the null model against all of the alternatives in (2) would be in terms of the hypotheses $H_{0}: \delta=0$ against $H_{1}: \delta \neq 0$.

Since spatial lags of the dependent variable appear in (4) our suggested procedure involves instrumental variables. For $i=1, \ldots, G$ let

$$
\begin{aligned}
H_{n}= & \left(X_{n}, W_{n} X_{n}, \ldots, W_{n}^{r} X_{n}, M_{n} X_{n}, M_{n} W_{n} X_{n}, \ldots, M_{n} W_{n}^{r} X_{n}\right)_{L I} \\
H_{n, i}= & \left(X_{n, i}, W_{n, i} X_{n, i}, \ldots, W_{n, i}^{r} X_{n, i}, M_{n, i} X_{n, i}, M_{n, i} W_{n, i} X_{n, i}\right. \\
& \left.\ldots, M_{n, i} W_{n, i}^{r} X_{n, i}\right)_{L I} \\
A_{n}= & \left(\bar{X}_{n}, W_{n} \bar{X}_{n}, \ldots, W_{n}^{r} \bar{X}_{n}, M_{n} \bar{X}_{n}, M_{n} W_{n} \bar{X}_{n}, \ldots, M_{n} W_{n}^{r} \bar{X}_{n}\right)_{L I}
\end{aligned}
$$

where $\bar{X}_{n}=\left(X_{n}, X_{n, 1} \ldots, X_{n, G}\right)$, the subscript $L I$ denotes the linearly independent columns of the indicated matrices, ${ }^{4}$ and, typically, one would take $r \leq 2$. The instrument matrices $H_{n}, H_{n, i}$, and $A_{n}$ are assumed to, at least, contain the linearly independent columns of, respectively $\left(X_{n}, M_{n} X_{n}\right)$, $\left(X_{n, i}, M_{n, i} X_{n, i}\right)$, and $\left(\bar{X}_{n}, M_{n} \bar{X}\right), i=1, \ldots, G$. Given this notation our suggested procedure is outlined below.

Step 1; Estimate the null model in (1) by two-stage least squares (henceforth 2SLS) using the instrument matrix $H_{n}$. Obtain the estimated residuals

\footnotetext{
${ }^{4}$ Among other things, in practice, at least some of the regressor matrices $X_{n, i}, i=$ $1, \ldots, G$ would have variables in common.
} 
from the null model, say $\hat{u}_{n}$. Also estimate the $i^{\text {th }}$ alternative model in (2) by 2 SLS using the instrument matrix $H_{n, i}, i=1, \ldots, G$.

Step 2: Take $\hat{\gamma}_{i, n}$ appearing in (4) as the 2SLS estimator based on $H_{n, i}$ of the $i^{\text {th }}$ alternative model, $\mathrm{i}=1, \ldots, \mathrm{G}$.

Step 3: Use the estimated residuals from the null model, namely $\hat{u}_{n}$, to estimate the parameter $\rho$ in the null model by the GMM procedure suggested in Kelejian and Prucha (1999). Denote this estimator as $\hat{\rho}_{n}$. Replace $\rho$ in (3) by $\hat{\rho}_{n}$ and estimate the resulting model by 2SLS using the instrument matrix $H_{n}$. This is the generalized spatial two stage least squares procedure suggested in Kelejian and Prucha (1998). Obtain the residual vector, say $\hat{\varepsilon}_{n}$, and use it to estimate the variance of the elements of the innovation vector: $\hat{\sigma}_{n, \varepsilon}^{2}=\hat{\varepsilon}_{n}^{\prime} \hat{\varepsilon}_{n} / n$.

Step 4: Replace $\rho$ in (4) by $\hat{\rho}_{n}$ and let

$$
\begin{aligned}
F_{n} & =\left(Z_{n, 1} \hat{\gamma}_{n, 1}, \ldots, Z_{n, G} \hat{\gamma}_{n, G}, M_{n, 1} Z_{n, 1} \hat{\gamma}_{n, 1}, \ldots, M_{n, G} Z_{n, G} \hat{\gamma}_{n, G}\right) \\
\delta & =\left(\alpha_{1},,, . \alpha_{G}, \phi_{1}, \ldots, \phi_{G}\right)^{\prime} .
\end{aligned}
$$

Given this notation, the empirical counterpart to (4) would be ${ }^{5}$

$$
y_{n}\left(\hat{\rho}_{n}\right) \sim Z_{n}\left(\hat{\rho}_{n}\right) \gamma+F_{n} \delta+\varepsilon_{n}
$$

Step 5: Estimate (5) by 2SLS using the instrument matrix $A_{n} \cdot{ }^{6}$ Specifically, denote the regressor matrix corresponding to (5) as $S_{n}=\left(Z_{n}\left(\hat{\rho}_{n}\right), F_{n}\right)$, and the regression parameters as $\eta^{\prime}=\left(\gamma^{\prime}, \delta^{\prime}\right)$. Note that under the null model the true value of $\eta$ is $\eta_{0}$ where $\eta_{0}^{\prime}=\left(\gamma^{\prime}, 0\right)$. Let $\hat{S}_{n}=P_{n} S_{n} \equiv\left(\hat{Z}_{n}\left(\hat{\rho}_{n}\right), \hat{F}_{n}\right)$ where $P_{n}=A_{n}\left(A_{n}^{\prime} A_{n}\right)^{-1} A_{n}^{\prime}$. Then the 2SLS estimator of $\eta$ is

$$
\hat{\eta}_{n}=\left(\hat{S}_{n}^{\prime} \hat{S}_{n}\right)^{-1} \hat{S}_{n}^{\prime} y_{n}\left(\hat{\rho}_{n}\right)
$$

The proof of Theorem 1 is given in the appendix.

Theorem 1: Under Assumptions A.1-A.7 given in the appendix

$$
\begin{aligned}
& n^{1 / 2}\left(\hat{\eta}_{n}-\eta_{0}\right) \stackrel{D}{\rightarrow} N\left(0, \sigma_{\varepsilon}^{2} p \lim _{n \rightarrow \infty} n\left(\hat{S}_{n}^{\prime} \hat{S}_{n}\right)^{-1}\right) \\
& \hat{\sigma}_{n, \varepsilon}^{2} \stackrel{P}{\rightarrow} \sigma_{\varepsilon}^{2}
\end{aligned}
$$

\footnotetext{
${ }^{5}$ The model in (5) is not exact, even if $\delta=0$ because $\hat{\rho}_{n} \neq \rho$.

${ }^{6}$ The large sample results described below will go through if at this stage the instrument matrix $A_{n}$ is replaced by $H_{n}$. However, one suspects that the power of the test will increase if the instrument matrix is taken to be $A_{n}$. This issue deserves further attention, especially if $G$ is large!
} 
Small sample inferences can be based on the approximation

$$
\hat{\eta}_{n} \simeq N\left[\eta, \hat{\sigma}_{n, \varepsilon}^{2}\left(\hat{S}_{n}^{\prime} \hat{S}_{n}\right)^{-1}\right]
$$

Let $\bar{k}=k+1+2 G$ and, using evident notation let $\hat{\eta}_{n}^{\prime}=\left(\hat{\gamma}_{n}^{\prime}, \hat{\delta}_{n}^{\prime}\right)$; let $\hat{V}_{n, \hat{\delta}}$ be the estimated small sample variance-covariance matrix corresponding to $\hat{\delta}_{n}$ - i.e., the lower $2 G \times 2 G$ submatrix of the $\bar{k} \times \bar{k}$ matrix $\hat{\sigma}_{n, \varepsilon}^{2}\left(\hat{S}_{n}^{\prime} \hat{S}_{n}\right)^{-1}$. Then, a Wald test of $H_{0}: \delta=0$ against $H_{1}: \delta \neq 0$ at the $a \%$ level of significance would be to reject $H_{0}$ if

$$
\hat{\delta}_{n}^{\prime} \hat{V}_{n, \hat{\delta}}^{-1} \hat{\delta}_{n}>\chi_{1-a}^{2}(2 G)
$$

The test suggested in (9) can easily be carried out in most software packages.

Remark : It is reasonable to assume that researchers may want to estimate the autoregressive parameter $\rho_{i}$ in the alternative models given in (2) by the GMM approach of Kelejian and Prucha (1999). Denote these estimators as $\hat{\rho}_{n, i}, i=1, \ldots, G$. Under further assumptions, it is not difficult to show that if the null model is the true model, and $\hat{\rho}_{n, i}$ is the linear GMM estimator described in Kelejian and Prucha (1999), then $p \lim _{n \rightarrow \infty} \hat{\rho}_{n, i}=$ $c_{i}, i=1, \ldots, G$ where $c_{i}$ is a finite constant. We conjecture that the nonlinear GMM estimator of $\rho_{i}$ also converges to a finite constant under reasonable assumptions. If so, the suggested test can be based on the first line of (4), instead of the second line. That is, in that first line replace $\rho_{i}$ and $\rho$, by, respectively $\hat{\rho}_{n, i}, i=1, \ldots, G$, and $\hat{\rho}_{n}$ obtained in step 3 . That model would then be estimated by 2SLS in terms of the instruments $A_{n}$. One would then test the hypothesis $\alpha_{i}=0, \mathrm{i}=1, \ldots, \mathrm{G}$ in the usual way. Under these conditions it is not clear whether this procedure is efficient relative to the one suggested in reference to (9).

Remark 2: Space limitations prevent a formal analysis of an extension of our results to a panel data framework. However, we conjecture that, under reasonable assumptions, an obvious extension of our results to such a framework will hold. Specifically, in a panel data setting suppose the disturbance term in each time period is a spatial AR(1) with innovations specified as error components, as in Kapoor, Kelejian, and Prucha (2007). Suppose there are $\mathrm{G}$ alternative models of a similar sort. Then we suggest the following procedure. (a) Estimate the null model in terms of an instrumental variable procedure; also estimate the parameters of the disturbance process of that null model by the GMM procedure described in Kapoor, Kelejian, and Prucha (2007). (b) Use the estimated parameters of the disturbance 
process to transpose the null model into one which corresponds to a model which has an error term which is not spatially correlated, time autocorrelated, or heteroskedastic- see Kapoor, Kelejian, and Prucha (2007) for useful transformations. (c) Estimate the regression parameters of the alternative models, and use these estimated regression parameters to construct variables which reflect the predictive influence of the alternative models. Add these constructed variables to the transformed null model. (d) Estimate the resulting expanded and transformed null model by the 2SLS procedure. (e) Test the significance of the added variables in the usual way.

\section{A Appendix}

Assumption A.1 All diagonal elements of the spatial weighting matrices $W_{n}, M_{n}, W_{n, i}$ are zero, $i=1, \ldots, G$.

Assumption A.2 The matrices $\left(I-\lambda W_{n}\right)$ and $\left(I-\rho M_{n}\right)$ are nonsingular for all $|\lambda|<1$ and $|\rho|<1$.

Assumption A.3 The row and column sums of the matrices $W_{n}, M_{n}, W_{n, i}$, $M_{n, i},\left(I-\lambda W_{n}\right)^{-1}$, and $\left(I-\rho M_{n}\right)^{-1}$ are bounded uniformly in absolute value, $i=1, \ldots, G$.

Assumption A.4 The regressor matrices $X_{n}, X_{n, i}, i=1, \ldots, G$ have full column rank (for $n$ large enough). Furthermore, the elements of these matrices are uniformly bounded in absolute value.

Assumption A.5 The elements of the innovation vector $\varepsilon_{n}$ in the null model, namely $\left\{\varepsilon_{i, n}: 1 \leq i \leq n, n \geq 1\right\}$ are distributed identically. Further, the innovations $\left\{\varepsilon_{i, n}: 1 \leq i \leq n\right\}$ are for each $n$ distributed (jointly) independently with $E\left(\varepsilon_{i, n}\right)=0, E\left(\varepsilon_{i, n}^{2}\right)=\sigma_{\varepsilon}^{2}$, where $0<\sigma_{\varepsilon}^{2}<b$ with $b<\infty$. Additionally the innovations are assumed to possess finite fourth moments.

Assumption A.6 Let $S_{n}^{*}=\left[Z_{n}-\rho M_{n} Z_{n}, F_{n}^{*}\right]$, where $F_{n}^{*}$ is identical to $F_{n}$ except that $\hat{\gamma}_{n, i}$ is replaced by $p \lim \hat{\gamma}_{n, i}=\phi_{i}, i=1, \ldots, G{ }^{7}$ Then we assume that the instrument matrices satisfy:

(a)

$$
\begin{aligned}
Q_{H H} & =\lim _{n \rightarrow \infty} n^{-1} H_{n}^{\prime} H_{n} \\
Q_{H_{i} H_{i}} & =\lim _{n \rightarrow \infty} n^{-1} H_{n, i}^{\prime} H_{n, i}, i=1, \ldots, G \\
Q_{A A} & =\lim _{n \rightarrow \infty} n^{-1} A_{n}^{\prime} A_{n}
\end{aligned}
$$

\footnotetext{
${ }^{7}$ An expression for $\phi_{i}, i=1, \ldots, G$ is given in the appendix.
} 
where $Q_{H H}, Q_{H_{i} H_{i}}$, and $Q_{A A}$ are finite and nonsingular.

(b)

$$
\begin{aligned}
Q_{H Z} & =\operatorname{plim}_{n \rightarrow \infty} n^{-1} H_{n}^{\prime} Z_{n} \\
Q_{H_{i} Z_{i}} & =\operatorname{plim}_{n \rightarrow \infty} n^{-1} H_{n, i}^{\prime} Z_{n, i}, i=1, \ldots, G \\
Q_{H_{i} Z} & =\operatorname{plim}_{n \rightarrow \infty} n^{-1} H_{n, i}^{\prime} Z_{n}, i=1, \ldots, G \\
Q_{A S^{*}} & =\operatorname{plim}_{n \rightarrow \infty} n^{-1} A_{n}^{\prime} S_{n}^{*} \\
Q_{H M Z} & =\operatorname{plim}_{n \rightarrow \infty} n^{-1} H_{n}^{\prime} M_{n} Z_{n}
\end{aligned}
$$

where $Q_{H Z}, Q_{H_{i} Z_{i}}, Q_{H_{i} Z}, Q_{A S^{*}}$, and $Q_{H M Z}$, are finite matrices, and $Q_{H Z}, Q_{H_{i} Z_{i}}, Q_{A S^{*}}$, and $Q_{H M Z}$ have full column rank. Furthermore, the matrix

$$
Q_{H Z}-\rho Q_{H M Z}=\operatorname{plim}_{n \rightarrow \infty} n^{-1} H_{n}^{\prime}\left(I-\rho M_{n}\right) Z_{n}
$$

has full column rank for all $|\rho|<1$.

(c)

$$
\begin{aligned}
& \Phi_{H}=\lim _{n \rightarrow \infty} n^{-1} H_{n}^{\prime}\left(I-\rho M_{n}\right)^{-1}\left(I-\rho M_{n}^{\prime}\right)^{-1} H_{n} \\
& \Phi_{A}=\lim _{n \rightarrow \infty} n^{-1} A_{n}^{\prime}\left(I-\rho M_{n}\right)^{-1}\left(I-\rho M_{n}^{\prime}\right)^{-1} A_{n}
\end{aligned}
$$

where $\Phi_{H}$ and $\Phi_{A}$ are finite and nonsingular for all $|\rho|<1$.

The following assumption ensures that the autoregressive parameter $\rho$ in the null model is "identifiably unique", cp. Kelejian and Prucha (1999).

Assumption A.7 The smallest eigenvalue of $\Gamma_{n}^{\prime} \Gamma_{n}$ is bounded away from zero, i.e., $\lambda_{\min }\left(\Gamma_{n}^{\prime} \Gamma_{n}\right) \geq \lambda_{*}>0$, where

$$
\Gamma_{n}=\frac{1}{n}\left(\begin{array}{lll}
2 E\left(u_{n}^{\prime} \bar{u}_{n}\right) & -E\left(\bar{u}_{n}^{\prime} \bar{u}_{n}\right) & 1 \\
2 E\left(\overline{\bar{u}}_{n}^{\prime} \bar{u}_{n}\right) & -E\left(\overline{\bar{u}}_{n}^{\prime} \overline{\bar{u}}_{n}\right) & \operatorname{tr}\left(M_{n}^{\prime} M_{n}\right) \\
E\left(u_{n}^{\prime} \overline{\bar{u}}_{n}+\bar{u}_{n}^{\prime} \bar{u}_{n}\right) & -E\left(\bar{u}_{n}^{\prime} \overline{\bar{u}}_{n}\right) & 0
\end{array}\right)
$$

and $\bar{u}_{n}=M_{n} u_{n}$ and $\overline{\bar{u}}_{n}=M_{n} \bar{u}_{n}=M_{n}^{2} u_{n}$.

Proof of Theorem 1: Since Assumptions 1-8 in Kelejian and Prucha (1998) are contained in our list of assumptions, $\hat{\rho}_{n}$ and $\hat{\sigma}_{n}^{2}$ obtained from our null model in step 3 are consistent. Also, our Assumptions A.3, A.5, parts 
(a) and (b) of A.6, and A.7 imply $p \lim _{n \rightarrow \infty} \hat{\gamma}_{n, i} \stackrel{P}{\rightarrow} \phi_{i}, i=1, \ldots, G$ where $\phi_{i}$ is a $k_{i} \times 1$ vector of finite constants, namely

$$
\phi_{i}=\left[Q_{H_{i} Z_{i}}^{\prime} Q_{H_{i} H_{i}}^{-1} Q_{H_{i} Z_{i}}\right]^{-1} Q_{H_{i} Z_{i}}^{\prime} Q_{H_{i} H_{i}}^{-1} Q_{H_{i} Z} \gamma
$$

Let $\pi_{n, i}=n^{-1} H_{n, i}^{\prime} u_{n}$. Then underlying (A.2) is the result that $\pi_{n, i} \stackrel{P}{\rightarrow} 0$. This follows from Chebyshev's inequality since $E\left(\pi_{n, i}\right)=0$ and $\operatorname{VC}\left(\pi_{n, i}\right)=$ $\sigma_{\varepsilon}^{2} n^{-2} H_{n, i}^{\prime}\left(I_{n}-\rho M_{n}\right)\left(I_{n}-\rho M_{n}\right)^{\prime} H_{n, i} \rightarrow 0$ since via Assumptions A.3, A.4, and A.5 the elements of $n V C\left(\pi_{n, i}\right)$ are 0(1) - see Kelejian and Prucha (1999).

Since $\hat{\rho}_{n}$ is consistent, $\hat{\rho}_{n}=\rho+\Delta_{n}$ where $\Delta_{n} \stackrel{P}{\rightarrow} 0$. The null model (1) then implies

$$
\begin{aligned}
y_{n}\left(\hat{\rho}_{n}\right) & =Z_{n}\left(\hat{\rho}_{n}\right) \gamma+u_{n}-\hat{\rho}_{n} M_{n} u_{n} \\
& =Z_{n}\left(\hat{\rho}_{n}\right) \gamma+\varepsilon_{n}-\Delta_{n} M_{n} u_{n}
\end{aligned}
$$

where, consistent with earlier notation $y_{n}\left(\hat{\rho}_{n}\right)=y_{n}-\hat{\rho}_{n} M_{n} y_{n}$, etc. Given this notation, and noting that $\hat{Z}_{n}^{\prime}\left(\hat{\rho}_{n}\right) Z_{n}\left(\hat{\rho}_{n}\right)=\hat{Z}_{n}^{\prime}\left(\hat{\rho}_{n}\right) \hat{Z}_{n}\left(\hat{\rho}_{n}\right)$ and $\hat{F}_{n}^{\prime} Z_{n}\left(\hat{\rho}_{n}\right)=$ $\hat{F}_{n}^{\prime} \hat{Z}_{n}\left(\hat{\rho}_{n}\right)$, it follows that $\hat{\eta}_{n}$ in (6) can be expressed as

$$
\begin{aligned}
\hat{\eta}_{n} & =\left[\begin{array}{c}
\hat{\gamma}_{n} \\
\hat{\delta}_{n}
\end{array}\right] \\
& =\left[\begin{array}{cc}
\hat{Z}_{n}^{\prime}\left(\hat{\rho}_{n}\right) \hat{Z}_{n}\left(\hat{\rho}_{n}\right) & \hat{Z}_{n}^{\prime}\left(\hat{\rho}_{n}\right) \hat{F}_{n} \\
\hat{F}_{n}^{\prime} \hat{Z}_{n}\left(\hat{\rho}_{n}\right) & \hat{F}_{n}^{\prime} \hat{F}_{n}
\end{array}\right]^{-1}\left[\begin{array}{c}
\hat{Z}_{n}^{\prime}\left(\hat{\rho}_{n}\right) \\
\hat{F}_{n}^{\prime}
\end{array}\right]\left[Z_{n}(\hat{\rho}) \gamma+\varepsilon_{n}-\Delta_{n} M_{n} u_{n}\right] \\
& =\left[\begin{array}{c}
\gamma \\
0
\end{array}\right]+\left[\begin{array}{cc}
\hat{Z}_{n}^{\prime}\left(\hat{\rho}_{n}\right) \hat{Z}_{n}\left(\hat{\rho}_{n}\right) & \hat{Z}_{n}^{\prime}\left(\hat{\rho}_{n}\right) \hat{F}_{n} \\
\hat{F}_{n}^{\prime} \hat{Z}_{n}\left(\hat{\rho}_{n}\right) & \hat{F}_{n}^{\prime} \hat{F}_{n}
\end{array}\right]^{-1}\left[\begin{array}{c}
\hat{Z}_{n}^{\prime}\left(\hat{\rho}_{n}\right) \\
\hat{F}_{n}^{\prime}
\end{array}\right]\left[\varepsilon_{n}-\Delta_{n} M_{n} u_{n}\right]
\end{aligned}
$$

Therefore

$$
\begin{aligned}
n^{1 / 2}\left[\begin{array}{c}
\hat{\gamma}_{n-\gamma}-\gamma \\
\hat{\delta}_{n}
\end{array}\right]= & n\left[\begin{array}{cc}
\hat{Z}_{n}^{\prime}\left(\hat{\rho}_{n}\right) \hat{Z}_{n}\left(\hat{\rho}_{n}\right) & \hat{Z}_{n}^{\prime}\left(\hat{\rho}_{n}\right) \hat{F}_{n} \\
\hat{F}_{n}^{\prime} \hat{Z}_{n}\left(\hat{\rho}_{n}\right) & \hat{F}_{n} \hat{F}_{n}
\end{array}\right]^{-1} * \\
& n^{-1 / 2}\left[\begin{array}{c}
\hat{Z}_{n}^{\prime}\left(\hat{\rho}_{n}\right)\left(\varepsilon_{n}-\Delta_{n} M_{n} u_{n}\right) \\
\hat{F}_{n}^{\prime}\left(\varepsilon_{n}-\Delta_{n} M_{n} u_{n}\right)
\end{array}\right] \\
= & n\left(\hat{S}_{n}^{\prime} \hat{S}_{n}\right)^{-1} n^{-1 / 2}\left[\begin{array}{c}
\hat{Z}_{n}^{\prime}\left(\hat{\rho}_{n}\right)\left(\varepsilon_{n}-\Delta_{n} M_{n} u_{n}\right) \\
\hat{F}_{n}\left(\varepsilon_{n}-\Delta_{n} M_{n} u_{n}\right)
\end{array}\right]
\end{aligned}
$$

Given that $\hat{\rho}_{n} \stackrel{P}{\rightarrow} \rho$, and $\hat{\gamma}_{n, i} \stackrel{P}{\rightarrow} \phi_{i}, i=1, \ldots, G$, parts (a) and (b) of Assumption 6 relating to $Q_{A A}$ and $Q_{A S^{*}}$ imply

$$
n\left(\hat{S}_{n}^{\prime} \hat{S}_{n}\right)^{-1} \stackrel{P}{\rightarrow} Q_{\hat{S} \hat{S}}^{-1}=\left(Q_{A S^{*}}^{\prime} Q_{A A}^{-1} Q_{A S^{*}}\right)^{-1}
$$


where $Q_{\hat{S} \hat{S}}$ is an $(k+1+2 G) \times(k+1+2 G)$ finite nonsingular matrix.

Now note that in (A.5) the term $n^{-1 / 2} \hat{Z}_{n}^{\prime}\left(\hat{\rho}_{n}\right) M_{n} u_{n} \Delta_{n} \stackrel{P}{\rightarrow} 0$ since

$$
\begin{aligned}
n^{-1 / 2} \hat{Z}_{n}^{\prime}\left(\hat{\rho}_{n}\right) M_{n} u_{n} \Delta_{n}= & {\left[n\left(Z_{n}-\hat{\rho}_{n} M_{n} Z_{n}\right)^{\prime} A_{n}\right]\left[n\left(A_{n}^{\prime} A_{n}\right)^{-1}\right] * } \\
& {\left[n^{-1 / 2} A_{n}^{\prime} M_{n} u_{n} \Delta_{n}\right] } \\
& \stackrel{P}{\rightarrow} Q_{A,(Z-\rho M Z)}^{\prime} Q_{A A}^{-1}\left[n^{-1 / 2} A_{n}^{\prime} M_{n} u_{n} \Delta_{n}\right] \stackrel{P}{\rightarrow} 0
\end{aligned}
$$

where $Q_{A,(Z-\rho M Z)}$ is the first $k+1$ columns of the finite matrix $Q_{A S^{*}}$, and $n^{-1 / 2} A_{n}^{\prime} M_{n} u_{n} \Delta_{n} \stackrel{P}{\rightarrow} 0$ since the elements of $n^{-1 / 2} A_{n}^{\prime} M_{n} u_{n}$ are $0_{P}(1)$ and $\Delta_{n} \stackrel{P}{\rightarrow} 0$.

Still focusing on (A.5), we have in a similar fashion

$$
n^{-1 / 2} \hat{F}_{n}^{\prime} M_{n} u_{n} \Delta_{n}=\left[n^{-1} F_{n}^{\prime} A_{n}\right]\left[n\left(A_{n}^{\prime} A_{n}\right)^{-1}\right]\left[n^{-1 / 2} A_{n}^{\prime} M_{n} u_{n} \Delta_{n}\right] \stackrel{P}{\rightarrow} 0
$$

The results in (A.4)-(A.8) imply

$$
n^{1 / 2}\left[\begin{array}{c}
\hat{\gamma}_{n}-\gamma \\
\hat{\delta}_{n}
\end{array}\right]-Q_{\hat{S} \hat{S}}^{-1} n^{-1 / 2}\left[\begin{array}{c}
\hat{Z}_{n}^{\prime}\left(\hat{\rho}_{n}\right) \\
\hat{F}_{n}^{\prime}
\end{array}\right] \varepsilon_{n} \stackrel{P}{\rightarrow} 0
$$

or, recalling that $\hat{S}_{n}=A_{n}\left(A_{n}^{\prime} A_{n}\right)^{-1} A_{n}^{\prime}\left[Z_{n}(\hat{\rho}), F_{n}\right)$

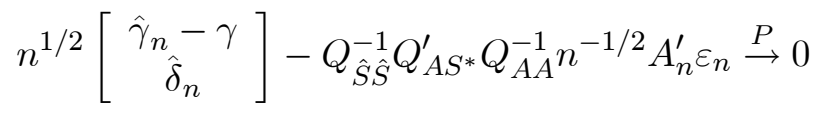

By Assumptions A.3 and A.4 the elements of $A_{n}$ are uniformly bound in absolute value, and by part (a) of Assumption A.6, $n^{-1} A_{n}^{\prime} A_{n}$ limits to a finite nonsingular matrix, $Q_{A A}$. Given this, and Assumption A.5 concerning the innovations, the central limit theorem described in Kelejian and Prucha (1998) implies

$$
n^{-1 / 2} A_{n}^{\prime} \varepsilon_{n} \stackrel{D}{\rightarrow} N\left(0, \sigma_{\varepsilon}^{2} Q_{A A}\right)
$$

The proof of Theorem 1 then follows from (A.10) and (A.11), namely

$$
\begin{aligned}
n^{1 / 2}\left[\begin{array}{c}
\hat{\gamma}_{n}-\gamma \\
\hat{\delta}_{n}
\end{array}\right]= & n^{1 / 2} \hat{\eta}_{n} \\
& \stackrel{D}{\rightarrow} N\left(0, \sigma_{\varepsilon}^{2} Q_{\hat{S} \hat{S}}^{-1}\right)
\end{aligned}
$$

since $Q_{\hat{S} \hat{S}}^{-1}=Q_{A S^{*}}^{\prime} Q_{A A}^{-1} Q_{A S^{*}}$. 


\section{References}

[1] Anselin, L., 1986. Non-Nested Tests on the Weight Structure in Spatial Autoregressive Models: Some Monte Carlo Results, Journal of regional Science 26, 267-284.

[2] Davidson, R. and MacKinnon, J., 1981. Several tests for Model Specification in the Presence of Alternative Hypotheses, Econometrica 49, 781-794.

[3] Godfrey, L., 1983. Testing Nonnested Models After Estimation by Instrumental variables or Least Squares, Econometrica 51, 355-366.

[4] Greene, W., 2003. Econometric Analysis (fifth edition), Prentice Hall, Upper Saddle River.

[5] Kelejian, H.H. and I.R. Prucha, 1998, A generalized spatial two-stage least squares procedure for estimating a spatial autoregressive model with autoregressive disturbances, Journal of Real Estate Finance and Economics 17, 99-121.

[6] Kelejian, H.H. and I.R. Prucha, 1999, A generalized moments estimator for the autoregressive parameter in a spatial model, International Economic Review 40, 509-533.

[7] Kapoor, M, Kelejian, H., and Prucha, I. 2007. Panel Data Models with Spatially Correlated Error Components, Journal of Econometrics 140, 97-130.

[8] Kmenta, J., 1986. Elements of Econometrics (second edition), Macmillan, New York.

[9] MacKinnon, J., White, H., and Davidson, R., 1983. Tests for Model Specification in the Presence of Alternative Hypotheses, Econometrica 21, 53-70.

[10] Pesaran, M. and Weeks, M., 2001. Nonnested Hypothesis Testing: An Overview, in A Companion to Theoretical Econometrics, Baltagi, B. (editor), Blackwell Publishers, Malden, Mass. 